Research Paper

\title{
Risk factors associated with Para-Aortic Lymph Node Failure after pelvic irradiation in patients with Cervical Cancer
}

\begin{abstract}
Weiping Wang ${ }^{*}$, Dunhuang Wang ${ }^{*}$, Xiaoliang Liu, Yuncan Zhou, Jiabin Ma, Xiaorong $\mathrm{Hou}^{凶}, \mathrm{Ke} \mathrm{Hu}^{\bowtie}$ and Fuquan Zhang

Department of Radiation Oncology, Peking Union Medical College Hospital, Chinese Academy of Medical Sciences \& Peking Union Medical College, Beijing, China.

*These authors contributed equally to this work.

$\square$ Corresponding authors: Xiaorong Hou, PhD, Department of Radiation Oncology, Peking Union Medical College Hospital, Chinese Academy of Medical Sciences \& Peking Union Medical College, No.1 Shuaifuyuan Wangfujing Dongcheng District, Beijing, China. Tel: +86-010-69155487. Fax: +86-010-69155487. E-mail: hxr_pumch@163.com; Ke Hu, MD, Department of Radiation Oncology, Peking Union Medical College Hospital, Chinese Academy of Medical Sciences \& Peking Union Medical College, No.1 Shuaifuyuan Wangfujing Dongcheng District, Beijing, China. Tel: +86-010-69155482. Fax: +86-10-6512-4875. E-mail: huk@pumch.cn.
\end{abstract}

() The author(s). This is an open access article distributed under the terms of the Creative Commons Attribution License (https://creativecommons.org/licenses/by/4.0/). See http:/ /ivyspring.com/terms for full terms and conditions.

Received: 2020.03.01; Accepted: 2020.06.14; Published: 2020.06.28

\begin{abstract}
Objective: Previous studies have shown that prophylactic extended-field irradiation can reduce para-aortic lymph node failure (PALNF) rates in patients with cervical cancer. As such, this type of irradiation may particularly benefit patients with a high risk of PALNF. In the present study, we analyzed the risk factors for PALNF in patients with cervical cancer treated with pelvic irradiation in order to identify potential indications of prophylactic extended-field irradiation.

Methods: We evaluated patients with 2018 FIGO stage IB3-IIICl cervical cancer who were treated with definitive pelvic radiotherapy or concurrent chemoradiotherapy at our institution between 2011 and 2014. Univariate and multivariate analyses were performed to identify risk factors for PALNF.

Results: We included 572 patients in the study. The median follow-up period was 37.9 months. Eighteen patients (3.1\%) first site of tumor relapse was the para-aortic lymph nodes, and thus showed PALNF. Using multivariate Cox regression analysis, we identified two significant risk factors for PALNF: tumor extension to the pelvic wall (hazard ratio, HR 3.60, $p=0.026$ ) and $\geq 2$ pelvic MLNs (HR 5.30, $p=0.005$ ). For patients with and without risk factors, the 3 -year overall survival, disease-free survival, and PALNF rates were $77.3 \%$ and $90.1 \%(p<0.001), 56.4 \%$ and $83.1 \%(p<0.001)$, and $12.0 \%$ and $2.3 \%(p<0.001)$, respectively.

Conclusion: Tumor extension to the pelvic wall and $\geq 2$ pelvic MLNs are positively associated with PALNF after pelvic irradiation in patients with cervical cancer. Further trials will be required to validate whether patients with these two risk factors may benefit from prophylactic extended-field irradiation.
\end{abstract}

Key words: cervical cancer; para-aortic lymph node failure; extended-field irradiation; indication

\section{Introduction}

Cervical cancer is the most common gynecological carcinoma in women worldwide [1]. In particular, locally advanced cervical cancer (LACC) accounts for a large proportion of patients in China, most likely because cervical cytologic screening methods have not been widely used in Chinese practice. The current standard treatment for patients with LACC is concurrent chemoradiotherapy (CCRT) globally. For LACC patients without para-aortic lymph node metastases, the standard radiation therapy treatment field is the pelvis. The para-aortic lymph node region is excluded from the treatment 
field, despite the fact that it plays an important role in cervical cancer metastasis and is one of the most common sites of tumor relapse $[2,3]$. To reduce the incidence of para-aortic lymph nodes failure (PALNF) and improve the survival of cervical cancer patients without para-aortic lymph node, para-aortic lymph nodes region is included in the target volume of radiation therapy in some institutes. This is prophylactic extended-field irradiation in patients with cervical cancer.

It is controversial whether prophylactic extended-field irradiation is beneficial for patients with LACC who were treated with CCRT [4-10]. In our previous study, extended-field irradiation decreased para-aortic lymph node failure (PALNF) and distant failure rates in patients with cervical cancer. However, overall survival (OS) and diseasefree survival (DFS) rates were not significantly different between prophylactic extended-field irradiation and pelvic radiotherapy, suggesting limited benefits of prophylactic extended-field irradiation [4]. One reason for this finding is that, in this previous study of ours, a large proportion of patients were early-stage patients without high-risk factors [4], and probably only patients with advanced disease could benefit from prophylactic extendedfield irradiation.

Considering prophylactic extended-field irradiation could reduce PALNF rates for patients with cervical cancer $[4,5,10]$, it may be of particular benefit to patients with a high risk of PALNF. Currently, studies on the risk factors for PALNF after pelvic irradiation in patients with cervical cancer are limited. A Taiwanese study of 758 patients with cervical cancer treated with pelvic irradiation found that, after a median follow-up of 50 months, 80 patients (11\%) showed PALNF. After the multivariate analysis, an SCC Ag level $\geq 40 \mathrm{ng} / \mathrm{ml}$, advanced parametrial involvement, and pelvic metastatic lymph nodes (MLNs) were identified as independent factors positively associated with PALNF. The 5-year PALNF rates in patients with SCC Ag $\geq 40 \mathrm{ng} / \mathrm{ml}$, parametrial score 4-6, pelvic MLNs, and no risk factor were $57 \%, 34 \%, 37 \%$, and $9 \%$, respectively[2]. In the present study, we analyzed the risk factors for PALNF in patients with cervical cancer treated with pelvic radiotherapy or CCRT in our institute to identify patients who may benefit from prophylactic extended-field irradiation.

\section{Materials and Methods}

\section{Patients}

We retrospectively reviewed the medical records of patients with cervical cancer treated with definitive radiotherapy or CCRT at our institute between January 2011 and December 2014. The inclusion criteria were as follows: biopsy-confirmed cervical cancer; 2018 International Federation of Gynecology and Obstetrics (FIGO) stage IB3 to IIIC1 [11]; and treatment with definitive pelvic radiation therapy or CCRT. Patients previously treated with extendedfield irradiation were excluded. The stage of patients was recorded with 2009 FIGO staging system [12] and converted to 2018 FIGO stage [11].

\section{Treatment}

The clinical target volume (CTV) for radiotherapy comprised the gross tumor, uterus, cervix, upper part of the vagina, parametrium and pelvic lymph node regions, with the latter comprising the common iliac, external iliac, internal iliac, obturator, and presacral lymph node regions. The upper border of the CTV was at the level of aortic bifurcation. The gross tumor volume (GTV) covered pelvic MLNs. The planning clinical target volume (PCTV) was defined as the CTV plus margins of 6-10 $\mathrm{mm}$. Margins of $5 \mathrm{~mm}$ were added to the GTV to form the planning gross tumor volume (PGTV). For intensity-modulated radiation therapy (IMRT), a dose of 50.4 Gy in 28 fractions was prescribed to the PCTV, and the PGTV was simultaneously boosted to 59-61 Gy. Image guidance was used for the delivery of IMRT [3]. Brachytherapy generally started after 3 weeks of IMRT. With high-dose-rate brachytherapy, 30-36 Gy in 5-7 fractions was delivered to point A. The first-line regimen of concurrent chemotherapy was 40 $\mathrm{mg} / \mathrm{m}^{2}$ cisplatin weekly. The detailed treatment protocol was described previously [4, 13].

\section{Follow-up}

Patients received a follow-up examination one month after the completion of treatment. Follow-up examinations were then conducted every three months during the first two years after treatment cessation, every six months during the next three years, and subsequently every year. These routine follow-up examinations included a gynecological examination, squamous cell carcinoma antigen (SCC Ag) measurement, pelvic MRI, and chest and abdominal CT. For patients with suspected tumor relapse, a tumor biopsy or positron emission tomography (PET)/CT was suggested.

\section{Statistics}

A univariate analysis was performed with logrank methods. A multivariate analysis was conducted with the Cox regression model. OS, DFS, and PALNF were calculated with Kaplan-Meier methods. Only the first site of relapse was evaluated. Tumor extension to the pelvic wall was diagnosed with gynecological 
examination, and the definition was the same with stage IIIB in 2009 FIGO staging system [11]. All statistical analyses were performed with SPSS (version 23.0; SPSS, Inc., Chicago, IL, USA).

\section{Results}

There were 723 patients with 2018 FIGO stage IB3-IIIC1 cervical cancer treated at our institute between January 2011 and December 2014. Of these patients, 151 were treated with extended-field irradiation, and were thus excluded. A total of 572 patients were therefore included in the present study. The basic demographic characteristics of the patients are shown in Table 1 . There were 48 patients $(8.4 \%)$ with tumor extension to the pelvic wall and 95 patients $(16.6 \%)$ with FIGO stage IIIC1 disease. Common iliac lymph nodes metastasis was observed in 5 patients $(0.9 \%)$.

The median follow-up period was 37.9 months (range, 1.0-76.2 months). The 3-year OS, DFS, and PALNF rates were $88.3 \%, 79.2 \%$, and $3.6 \%$ (Figure 1), respectively. Eighteen patients $(3.1 \%)$ experienced PALNF. The median time from the end of treatment to identification of PALNF was 14.0 months (range, 4.7-35.7 months). Of these 18 patients, 10 had only PALNF, while the other 8 had tumor relapse at other sites as well - 3 patients with pelvic lymph node relapse, 1 patient with vaginal recurrence, 1 patient with parametrial recurrence, 1 patient with lung metastasis, 1 patient with mediastinum metastasis and 1 patient with supraclavicular metastasis.

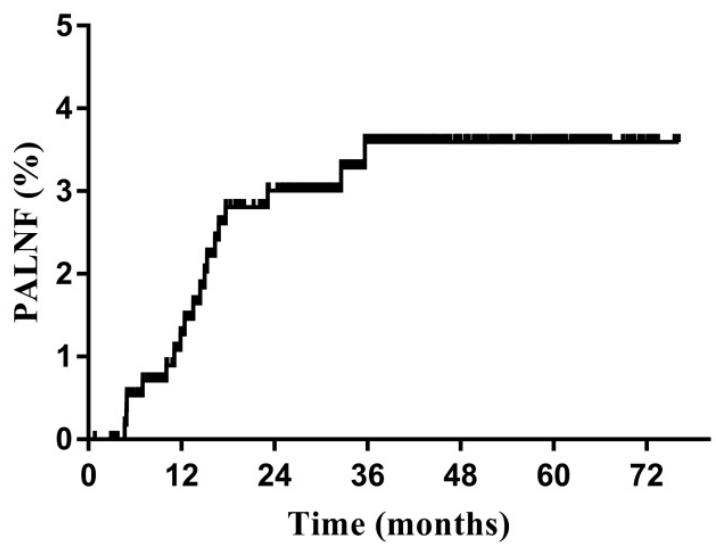

Figure 1. Para-aortic lymph node failure (PALNF) rates of patients with cervical cancer treated with pelvic irradiation $(n=572)$.

The univariate analysis of entire patient population revealed positive associations with PALNF for the 2018 FIGO stage of the tumor $(p<0.001)$, tumor extension to the pelvic wall $(p=0.015)$, the presence of common iliac MLNs $(p=0.026)$ and the number of pelvic MLNs $(p=0.001)$ (Table 2). Considering that tumor extension to the pelvic wall and the number of pelvic MLNs (0 vs. 1 vs.
22) were strongly correlated with 2018 FIGO stage (IB3-IIIA vs. IIIB vs. IIIC1), 2018 FIGO stage was not incorporated into the multivariate analysis. The multivariate analysis revealed two significant risk factors for PALNF: tumor extension to the pelvic wall (hazard ratio (HR) 3.60, 95\% confidence interval, CI 1.17-11.10, $\mathrm{p}=0.026$ ) and $\geq 2$ pelvic MLNs (HR 5.30, 95\% CI, 1.65-17.01, p=0.005) (Table 3).

Table 1. Baseline demographic, clinical and treatment characteristics of patients with 2018 FIGO stage IB3-IIICI cervical cancer treated with pelvic radiation therapy with or without concurrent chemotherapy

\begin{tabular}{|c|c|c|}
\hline Characteristic & Number of patients & $\begin{array}{l}\text { Percentage } \\
\text { of patients }\end{array}$ \\
\hline \multicolumn{3}{|l|}{ Age (years) } \\
\hline Median & 52 (range, 26-88) & \\
\hline$<65$ & 508 & $88.8 \%$ \\
\hline$\geq 65$ & 64 & $11.2 \%$ \\
\hline \multicolumn{3}{|l|}{ Histology } \\
\hline Squamous cell carcinoma & 506 & $88.5 \%$ \\
\hline Adenocarcinoma & 52 & $9.1 \%$ \\
\hline Adenosquamous cell carcinoma & 10 & $1.7 \%$ \\
\hline Others & 4 & $0.7 \%$ \\
\hline \multicolumn{3}{|l|}{2018 FIGO stage } \\
\hline IB3 & 35 & $6.1 \%$ \\
\hline IIA & 50 & $8.7 \%$ \\
\hline IIB & 335 & $58.6 \%$ \\
\hline IIIA & 18 & $3.1 \%$ \\
\hline IIIB & 39 & $6.8 \%$ \\
\hline IIIC1 & 95 & $16.6 \%$ \\
\hline \multicolumn{3}{|l|}{ Primary tumor size } \\
\hline$<4 \mathrm{~cm}$ & 227 & $39.7 \%$ \\
\hline$\geq 4 \mathrm{~cm}$ & 345 & $60.3 \%$ \\
\hline \multicolumn{3}{|c|}{ Tumor extension to the pelvic wall* } \\
\hline Yes & 48 & $8.4 \%$ \\
\hline No & 524 & $91.6 \%$ \\
\hline \multicolumn{3}{|l|}{ Common iliac MLNs } \\
\hline Yes & 5 & $0.9 \%$ \\
\hline No & 567 & $99.1 \%$ \\
\hline \multicolumn{3}{|l|}{ Bilateral pelvic MLNs } \\
\hline Yes & 37 & $6.5 \%$ \\
\hline No & 535 & $93.5 \%$ \\
\hline \multicolumn{3}{|l|}{ Number of pelvic MLNs } \\
\hline 0 & 477 & $83.4 \%$ \\
\hline 1 & 51 & $8.9 \%$ \\
\hline 2 & 36 & $6.3 \%$ \\
\hline$\geq 3$ & 8 & $1.4 \%$ \\
\hline \multicolumn{3}{|l|}{ Large pelvic MLNs ( $\geq 1.5 \mathrm{~cm}$ ) } \\
\hline Yes & 541 & $94.6 \%$ \\
\hline No & 31 & $5.4 \%$ \\
\hline \multicolumn{3}{|l|}{ Concurrent chemotherapy } \\
\hline Yes & 484 & $84.6 \%$ \\
\hline No & 88 & $15.4 \%$ \\
\hline
\end{tabular}

Having identified them as two key risk factors for PALNF, we next examined OS, DFS, and PALNF according to tumor extension to the pelvic wall and the number of pelvic MLNs. Tumor extension to the pelvic wall and $\geq 2$ pelvic MLNs were observed in 44 and 48 patients, respectively. For patients with and without tumor extension to the pelvic wall, the 3-year OS, DFS, and PALNF rates were $77.3 \%$ and $89.3 \%$ 
$(\mathrm{p}=0.003), 61.9 \%$ and $80.7 \%(\mathrm{p}<0.001)$, and $12.8 \%$ and $3.0 \%(p=0.015$, Figure 2$)$, respectively. For patients with $0-1$ pelvic MLNs versus $\geq 2$ pelvic MLNs, the 3 -year OS, DFS, and PALNF rates were $89.4 \%$ and $73.8 \%(\mathrm{p}<0.001), 82.1 \%$ and $45.5 \%(\mathrm{p}<0.001)$, and $2.8 \%$ and $14.9 \%(\mathrm{p}<0.001$, Figure 3$)$, respectively. There were 486,80 , and 6 patients with none, one, or both of the defined risk factors, respectively. For patients with and without risk factors, the 3-year OS, DFS, and PALNF rates were $77.3 \%$ and $90.1 \%(\mathrm{p}<0.001$, Figure $4 \mathrm{~A}), 56.4 \%$ and $83.1 \%(\mathrm{p}<0.001$, Figure $4 \mathrm{~B})$, and $12.0 \%$ and $2.3 \%(p<0.001$, Figure $4 \mathrm{C})$, respectively. For patients with one versus both risk factors, the 3-year OS, DFS, and PALNF rates were $79.3 \%$ and $50.0 \%$ $(\mathrm{p}=0.050), 60.1 \%$ and $16.7 \%(\mathrm{p}=0.013)$, and $10.1 \%$ and $50.0 \%(\mathrm{p}=0.286)$, respectively.

\section{Discussion}

In the present study, tumor extension to the pelvic wall and $\geq 2$ pelvic MLNs were associated with PALNF after pelvic irradiation in patients with LACC. Patients with these two factors may benefit from prophylactic EFI. These two identified risk factors for PALNF should be validated whether they are indeed predictive of the therapeutic success of prophylactic extended-field irradiation in patients with cervical cancer.

In our previous study, 133 patients with 2018 FIGO stage IIIB cervical cancer and treated with CCRT or radiotherapy were retrospectively analyzed. The 5-year OS and DFS of patients treated with pelvic radiotherapy and prophylactic extended-field irradiation were $66.3 \%$ and $80.3 \%(p=0.013), 57.2 \%$ and $80.4 \% \quad(p=0.002)$, respectively, suggesting that patients with 2018 FIGO stage IIIB cervical cancer could benefit from cervical cancer[14]. It should be noted that tumor extension to the pelvic wall was not the same with stage 2018 FIGO stage IIIB. With 2018 FIGO staging system, patients with pelvic lymph nodes metastasis was classified to IIIC1. In the present study, part of the patients with tumor extension to the pelvic wall had positive pelvic lymph nodes. In the study of Lee et al, 206 patients with LACC and negative para-aortic lymph nodes who underwent pelvic radiotherapy (110 patients) and prophylactic extended-field irradiation (96 patients) were retrospectively reviewed. In subgroup analysis, compared with pelvic irradiation, prophylactic extended-field irradiation improved the OS of patients with FIGO III-IVA. In this study, most patients (32/47) with FIGO stage III-IVA cervical cancer had stage IIIB disease, indicating patients with FIGO stage IIIB cervical cancer could benefit from prophylactic extended-field irradiation [6].
Table 2. Results of the univariate analysis for patients with cervical cancer treated with pelvic irradiation

\begin{tabular}{llc}
\hline Characteristic & 3 -year PALNF rate & P value \\
\hline $\begin{array}{l}\text { Age (years) } \\
<65\end{array}$ & $3.8 \%$ & \\
$\geq 65$ & $1.6 \%$ & \\
Histology & & \\
Squamous cell carcinoma & $3.6 \%$ & 0.901 \\
Nonsquamous cell carcinoma & $3.2 \%$ & \\
2018 FIGO stage & & \\
IB3-IIIA & $1.7 \%$ & $<0.001$ \\
IIIB & $9.5 \%$ & \\
IIIC1 & $11.3 \%$ & \\
Primary tumor size & & \\
$<4$ cm & $1.8 \%$ & 0.108 \\
$\geq 4$ cm & $4.8 \%$ & \\
Tumor extension to the pelvic wall & & \\
Yes & $12.8 \%$ & 0.015 \\
No & $3.0 \%$ & \\
Common iliac MLNs & & \\
Yes & $3.4 \%$ & \\
No & $20.0 \%$ & \\
Number of pelvic MLNs & & \\
0 & $2.2 \%$ & 0.001 \\
1 & $8.8 \%$ & \\
$\geq 2$ & $14.9 \%$ & 0.245 \\
Large pelvic MLNs ( 1.5 cm) & & \\
Yes & $11.2 \%$ & \\
No & $3.3 \%$ & \\
Concurrent chemotherapy & & \\
Yes & $3.8 \%$ & \\
No & $2.4 \%$ & \\
Abbreviations: MLNs = metastatic lymph nodes. & \\
\end{tabular}

Table 3. Results of the multivariate analysis for patients with cervical cancer treated with pelvic irradiation

\begin{tabular}{|c|c|c|}
\hline & PALNF & \\
\hline Variable & HR $(95 \% \mathrm{CI})$ & P value \\
\hline \multicolumn{3}{|c|}{ Tumor extension to the pelvic wall } \\
\hline No & Reference & 0.026 \\
\hline Yes & $3.60(1.17-11.10)$ & \\
\hline \multicolumn{3}{|c|}{ Common iliac MLNs } \\
\hline No & Reference & \\
\hline Yes & $2.30(0.25-21.21)$ & 0.461 \\
\hline \multicolumn{3}{|c|}{ Number of pelvic MLNs } \\
\hline 0 & Reference & \\
\hline 1 & $2.86(0.79-10.39)$ & 0.111 \\
\hline$\geq 2$ & $5.30(1.65-17.01)$ & 0.005 \\
\hline
\end{tabular}

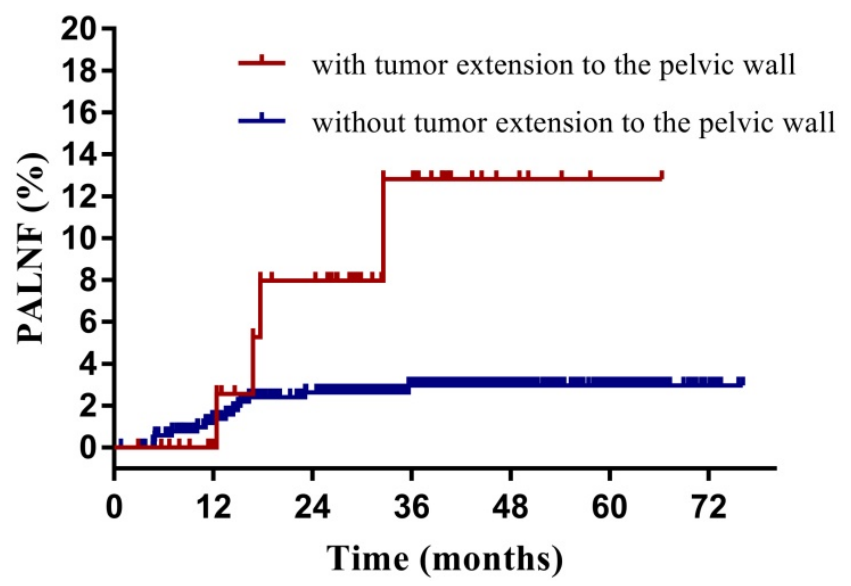

Figure 2. Para-aortic lymph node failure rates of cervical cancer patients with $(n=48)$ and without tumor extension to the pelvic wall $(n=524)$. 


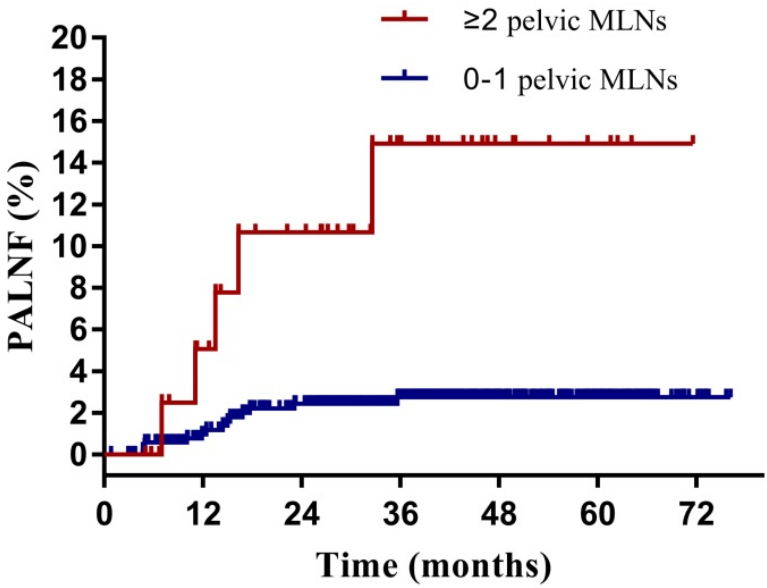

Figure 3. Para-aortic lymph node failure rates of cervical cancer patients with 0-1 $(n=528)$ or $\geq 2$ pelvic metastatic lymph nodes (MLNs, $n=44)$.

It was reported that pelvic MLNs was associated with PALNF after pelvic radiotherapy in patients with cervical cancer [2], and prophylactic extended-

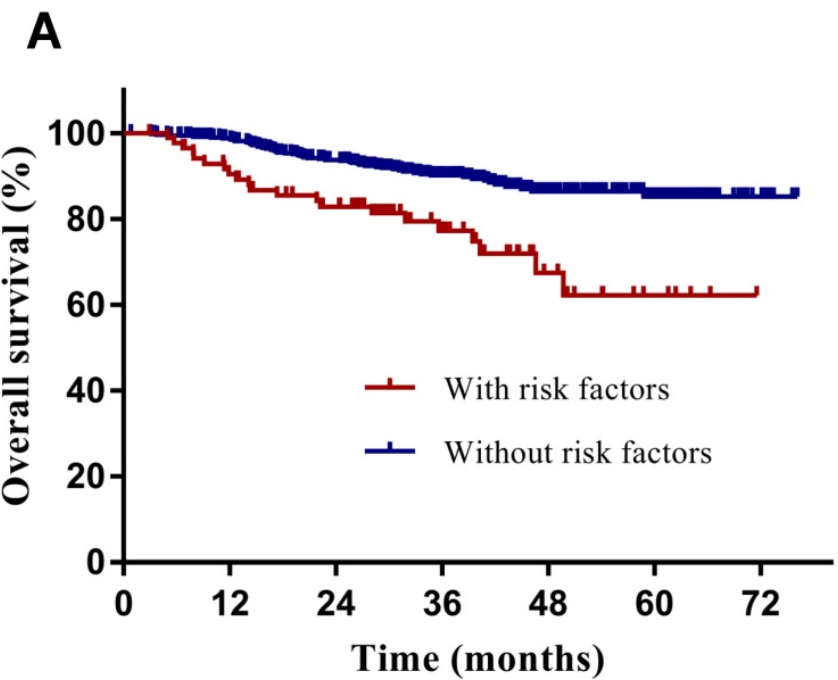

field irradiation improved the OS of patients with pelvic MLNs [6]. Studies focused on the number of pelvic MLNs and prophylactic extended-field irradiation was limited. In another study of Lee et al, 198 patients with LACC, pelvic MLNs and negative para-aortic lymph nodes were analyzed. Compared with pelvic radiotherapy, prophylactic extended-field irradiation significantly improved 5-year cancerspecific survival (56.5\% vs. $96.3 \%$, p<0.001) among patients with common iliac MLNs or $\geq 3$ pelvic MLNs. For patients with pelvic MLNs below the common iliac bifurcation and 1-2 pelvic MLNs, the cancerspecific survival was not significantly different [15]. In the present study, $\geq 2$ pelvic MLNs was a risk factor of PALNF after pelvic irradiation. As we know, this factor has not been validated predictive of the therapeutic success of prophylactic extended-field irradiation.
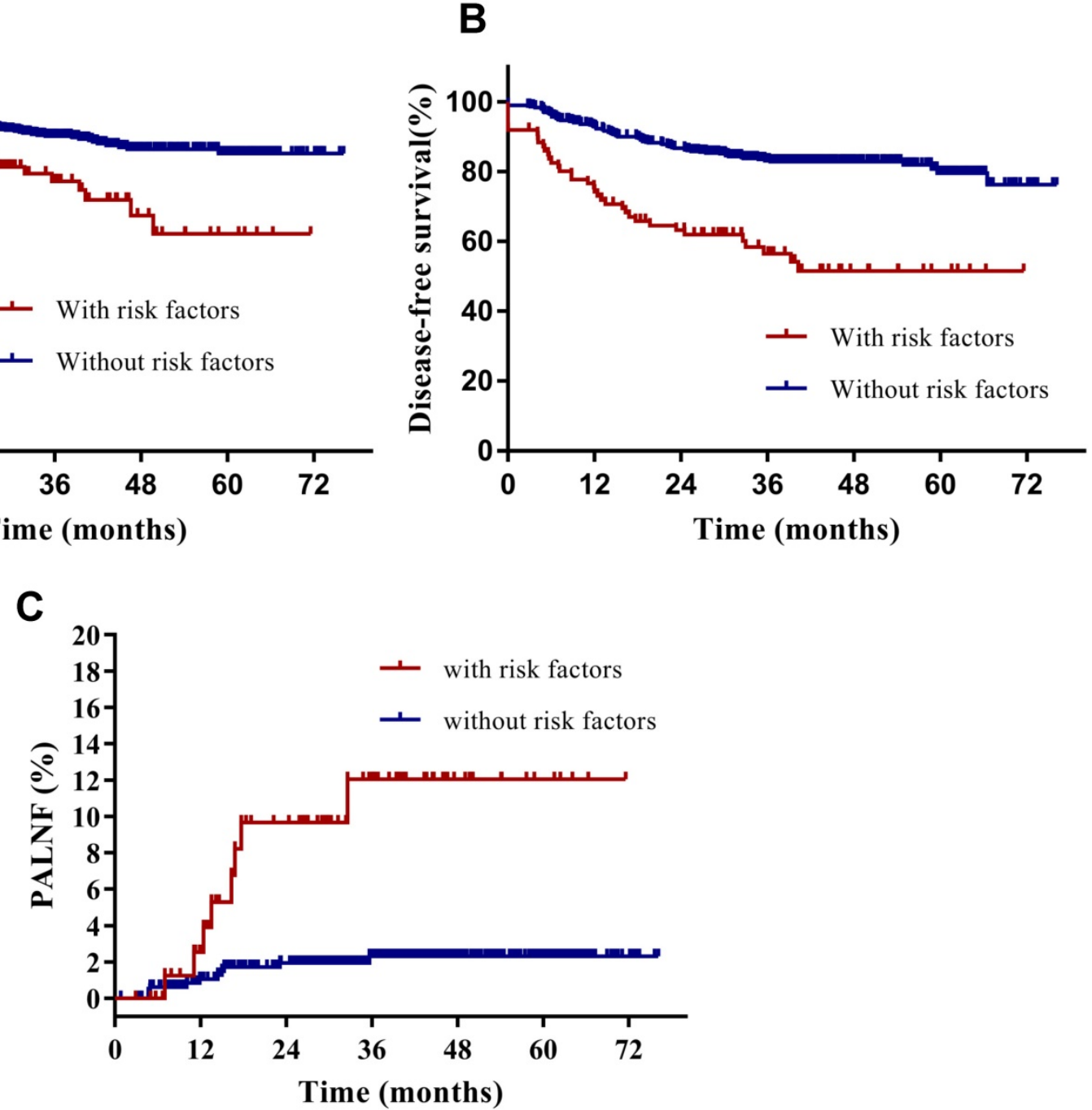

Figure 4. Disease-free survival (A), overall survival $(\mathbf{B})$ and para-aortic lymph node failure $(\mathbf{C})$ rates of cervical cancer patients with $(\mathrm{n}=86)$ and without identified risk factors $(n=486)$ for PALNF. 
We found that common iliac MLNs were not significantly associated with PALNF in patients with cervical cancer, likely because only 5 patients $(0.9 \%)$ in our cohort presented with them. However, it has been previously reported that common iliac MLNs are associated with para-aortic MLNs [16, 17]. Prophylactic extended-field irradiation improved the survival of patients with common iliac MLNs [15], and indeed the National Comprehensive Cancer Network (NCCN) guidelines also recommend that prophylactic extended-field irradiation be conducted for patients with common iliac MLNs. Therefore, common iliac MLNs are a clear indication of prophylactic extended-field irradiation, although our data could not draw that conclusion.

Tumor extensions to the pelvic wall and pelvic MLNs have been discussed extensively in patients with cervical cancer. It has been reported that these two factors are associated with poor survival in patients with cervical cancer [18-20]. In the present study, only patients without para-aortic MLNs who were treated with pelvic radiotherapy were included. We found that tumor extension to the pelvic wall and $\geq 2$ pelvic MLNs were associated with PALNF. This may potentially add some insight to the existing knowledge to this field.

The present study had some limitations. First, most patients with risk factors for PALNF had already received prophylactic extended-field irradiation and were therefore excluded from the study. As a result, compared with that of the primary population [4], the percentage of patients with risk factors for PALNF was small. The number of events was also limited in the present study, which may have influenced the study conclusions. With such a small sample size, especially the small sample size in some subgroups, it is difficult to draw a conclusive statistical statement. In addition, the conclusion of the present study should be interpreted with caution. Second, we did not validate whether the two identified risk factors for PALNF are indeed predictive of the therapeutic success of prophylactic extended-field irradiation in patients with cervical cancer in the present study. Further studies will be required to assess whether this hypothesized predictive potential is accurate in an independent cohort, such as publicly available databases. In 2019, our institute started a multicenter, randomized, phase 3 trial comparing pelvic radiotherapy and prophylactic EFI in selected patients with cervical cancer treated with concurrent chemoradiotherapy in China (NCT03955367). In this trial, tumor extension to the pelvic wall and $\geq 2$ pelvic MLNs was used as inclusion criteria.

In conclusion, tumor invasion of the pelvic wall and $\geq 2$ pelvic MLNs are related to PALNF after pelvic irradiation in patients with cervical cancer. If verified in subsequent clinical trials, these associations may inform the determination of which patients may benefit from prophylactic extended-field irradiation.

\section{Acknowledgements}

\section{Funding}

This study is supported by Key Technologies Research and Development Program of China (grant number 2016YFC0105207) and National Natural Science Foundation of China (grant number U19A2064).

\section{Competing Interests}

The authors have declared that no competing interest exists.

\section{References}

1. Bray F, Ferlay J, Soerjomataram I, Siegel RL, Torre LA, Jemal A. Global cancer statistics 2018: GLOBOCAN estimates of incidence and mortality worldwide for 36 cancers in 185 countries. CA Cancer J Clin. 2018; 68: 394-424.

2. Huang EY, Wang CJ, Chen HC, Fang FM, Huang YJ, Wang CY, et al. Multivariate analysis of para-aortic lymph node recurrence after definitive radiotherapy for stage IB-IVA squamous cell carcinoma of uterine cervix. Int J Radiat Oncol Biol Phys. 2008; 72: 834-42.

3. Wang W, Zhang F, Hu K, Hou X. Image-guided, intensity-modulated radiation therapy in definitive radiotherapy for 1433 patients with cervical cancer. Gynecol Oncol. 2018; 151: 444-8.

4. Wang W, Liu X, Meng Q, Zhang F, Hu K. Prophylactic Extended-Field Irradiation for Patients With Cervical Cancer Treated With Concurrent Chemoradiotherapy: A Propensity-Score Matching Analysis. Int J Gynecol Cancer. 2018; 28: 1584-91.

5. Asiri MA, Tunio MA, Mohamed R, Bayoumi Y, Alhadab A, Saleh RM, et al. Is extended-field concurrent chemoradiation an option for radiologic negative paraaortic lymph node, locally advanced cervical cancer? Cancer Manag Res. 2014; 6: 339-48.

6. Lee J, Lin JB, Chang CL, Jan YT, Sun FJ, Wu MH, et al. Prophylactic lower para-aortic irradiation using intensity-modulated radiotherapy mitigates the risk of para-aortic recurrence in locally advanced cervical cancer: A 10-year institutional experience. Gynecol Oncol. 2017; 146: 20-6.

7. Oh J, Seol KH, Lee HJ, Choi YS, Park JY, Bae JY. Prophylactic extended-field irradiation with concurrent chemotherapy for pelvic lymph node-positive cervical cancer. Radiation oncology journal. 2017; 35: 349-58.

8. Yap ML, Cuartero J, Yan J, Pintilie M, Fyles A, Levin W, et al. The role of elective para-aortic lymph node irradiation in patients with locally advanced cervical cancer. Clin Oncol (R Coll Radiol). 2014; 26: 797-803.

9. Park SG, Kim JH, Oh YK, Byun SJ, Kim MY, Kwon SH, et al. Is Prophylactic Irradiation to Para-aortic Lymph Nodes in Locally Advanced Cervical Cancer Necessary? Cancer Res Treat. 2014; 46: 374-82.

10. Kim JH, Kim JY, Yoon MS, Kim YS, Lee JH, Kim HJ, et al. Prophylactic irradiation of para-aortic lymph nodes for patients with locally advanced cervical cancers with and without high CA9 expression (KROG 07-01): A randomized, open-label, multicenter, phase 2 trial. Radiother Oncol. 2016; 120: 383-9.

11. Bhatla N, Aoki D, Sharma DN, Sankaranarayanan R. Cancer of the cervix uteri. Int J Gynaecol Obstet. 2018; 143 Suppl 2: 22-36.

12. Pecorelli S. Revised FIGO staging for carcinoma of the vulva, cervix, and endometrium. Int J Gynaecol Obstet. 2009; 105: 103-4.

13. Wang W, Hou X, Yan J, Shen J, Lian X, Sun S, et al. Outcome and toxicity of radical radiotherapy or concurrent Chemoradiotherapy for elderly cervical cancer women. BMC Cancer. 2017; 17: 510.

14. Meng Q, Liu X, Wang W, Hou X, Lian X, Sun S, et al. Evaluation of the efficacy of prophylactic extended field irradiation in the concomitant chemoradiotherapy treatment of locally advanced cervical cancer, stage IIIB in the 2018 FIGO classification. Radiat Oncol. 2019; 14: 228.

15. Lee J, Lin JB, Chang CL, Sun FJ, Wu MH, Jan YT, et al. Impact of para-aortic recurrence risk-guided intensity-modulated radiotherapy in 
locally advanced cervical cancer with positive pelvic lymph nodes. Gynecol Oncol. 2018; 148: 291-8.

16. Huang H, Liu J, Li Y, Wan T, Feng Y, Li Z, et al. Metastasis to deep obturator and para-aortic lymph nodes in 649 patients with cervical carcinoma. Eur J Surg Oncol. 2011; 37: 978-83.

17. Sakuragi N, Satoh C, Takeda N, Hareyama H, Takeda M, Yamamoto R, et al. Incidence and distribution pattern of pelvic and paraaortic lymph node metastasis in patients with Stages IB, IIA, and IIB cervical carcinoma treated with radical hysterectomy. Cancer. 1999; 85: 1547-54.

18. Kang S, Nam BH, Park JY, Seo SS, Ryu SY, Kim JW, et al. Risk assessment tool for distant recurrence after platinum-based concurrent chemoradiation in patients with locally advanced cervical cancer: a Korean gynecologic oncology group study. J Clin Oncol. 2012; 30: 2369-74.

19. Rose PG, Java J, Whitney CW, Stehman FB, Lanciano R, Thomas GM, et al. Nomograms Predicting Progression-Free Survival, Overall Survival, and Pelvic Recurrence in Locally Advanced Cervical Cancer Developed From an Analysis of Identifiable Prognostic Factors in Patients From NRG Oncology/Gynecologic Oncology Group Randomized Trials of Chemoradiotherapy. J Clin Oncol. 2015; 33: 2136-42.

20. Lei X, Qian $C Y$, Qing $Y$, Zhao KW, Yang ZZ, Dai N, et al. Californium-252 brachytherapy combined with external-beam radiotherapy for cervical cancer: long-term treatment results. Int J Radiat Oncol Biol Phys. 2011; 81: 1264-70. 